Trans

continentales
Transcontinentales

Sociétés, idéologies, système mondial

$12 / 13 \mid 2012$

Marchés de l'art émergents

\title{
Marché de l'art contemporain indien : territoires et réseaux en construction
}

Indian contemporary art market : territories and networks underconstruction

\section{Christine Ithurbide}

\section{(2) OpenEdition}

\section{Journals}

Édition électronique

URL : http://journals.openedition.org/transcontinentales/1347

DOI : $10.4000 /$ transcontinentales. 1347

ISBN : 978-2-7351-1599-0

ISSN : $1775-397 X$

\section{Éditeur}

Editions de la maison des sciences de l'homme

Référence électronique

Christine Ithurbide, "Marché de l'art contemporain indien : territoires et réseaux en construction », Transcontinentales [En ligne], 12/13 | 2012, document 6, mis en ligne le 30 août 2012, consulté le 08 septembre 2020. URL : http://journals.openedition.org/transcontinentales/1347 ; DOI : https://doi.org/ 10.4000/transcontinentales. 1347

Ce document a été généré automatiquement le 8 septembre 2020

Tous droits réservés 


\title{
Marché de l'art contemporain indien : territoires et réseaux en construction
}

\author{
Indian contemporary art market : territories and networks underconstruction
}

\author{
Christine Ithurbide
}

L'émergence du marché de l'art contemporain indien s'inscrit dans un contexte de changements économiques, sociaux et artistiques en Inde mais aussi de redéfinition des rapports centre/périphérie dans la géographie de l'art contemporain et d'apparition de nouvelles centralités en Asie-Pacifique. Les infrastructures et les acteurs du marché de l'art en Inde et dans le monde ont joué un rôle essentiel dans le processus de reconnaissance nationale et internationale de cette scène. Mon étude propose d'analyser les origines, les enjeux et les conséquences de l'émergence de ce marché dans les dynamiques urbaines et économiques, et sur l'évolution des pratiques artistiques et curatoriales indiennes. Si mon approche a pour ambition de présenter les processus qui ont permis la construction d'une scène artistique «indienne» et l'insertion de ce pays dans le réseau des capitales artistiques internationales, elle questionnera aussi les limites d'une création artistique devenue fortement dépendante du marché, de la compétition et des modèles internationaux.

\section{Émergence du marché de l'art : de l'indépendance aux années 1990}

De la fin $d_{u} x_{\text {XIII }}{ }^{\mathrm{e}}$ au début $\mathrm{du} \mathrm{xx}^{\mathrm{e}}$ siècle, un nouveau réseau d'infrastructures artistiques, d'écoles d'art, de musées et de sociétés artistiques ${ }^{1}$ se met en place progressivement sur le territoire indien. Il se construit grâce à la collaboration entre les Britanniques et plusieurs industriels et intellectuels indiens, dont Sir Jamsetjee Jeejeebhoy et Dr Bhau Daji Lad. Au lendemain de l'Indépendance, en 1947, le gouvernement indien inaugure différents espaces culturels qui ont pour vocation de 
participer à la naissance d'une identité artistique indienne postcoloniale. D'importantes galeries publiques sont créées à Delhi (AIFACS - All India Fine Arts and Crafts Society -, Triveni Kala Sangam) et à Bombay² (Jehangir Art Gallery), accompagnées de deux National Gallery of Modern Art (NGMA) et de nouvelles écoles d'art présentes dans toutes les capitales régionales, les Lalit Kala Akademi (LKA). En parallèle, les premières galeries privées s'installent à proximité du centre financier et administratif. Dhoomi Mall, en 1936 et Kumar Gallery, en 1955, ouvrent à Delhi, suivies en 1963, à Bombay, par Chemould et Pundole Art Gallery dans le quartier qui deviendra le futur district artistique Kala Ghoda-Colaba. Attirés par cet ensemble d'écoles, de galeries publiques et privées, de nombreux artistes migrent vers les capitales régionales et forment plusieurs groupes, en particulier à Baroda, Bombay, Calcutta, Madras et Delhi. Au début des années 1950, un marché de l'art est déjà présent dans les centres urbains, soutenu par des mécènes et des collectionneurs d'art. Parmi ces acteurs pionniers, on cite souvent les grandes familles marchandes ou industrielles Birla et Tata, ainsi que d'autres personnalités scientifiques et philanthropes, Homi Bhabha, Jehanghir Nicholson ou encore Ebrahim Alkazi.

Dans les années 1980, Indira Gandhi entreprend des réformes politiques et économiques en faveur du développement d'une économie de marché. De nouvelles galeries sont créées principalement à Delhi, Bombay et Calcutta. L'organisation du marché de l'art est encore relativement informelle et repose sur de fortes structures familiales et communautaires. La plupart des galeries sont dirigées par des femmes issues de milieux aisés qui ont progressivement reçu la gestion du patrimoine artistique familial. Leur clientèle s'appuie généralement sur des réseaux communautaires, en particulier Parsis ou Marwaris, connus pour avoir une place importante dans l'industrie et le commerce. S'il arrivait aux artistes, souvent des amis proches de la famille, de venir discuter et peindre dans la galerie, peu à peu, les relations amicales entre les artistes et les clients laissent place à une attitude plus professionnelle ${ }^{3}$.

Cette période d'ouverture économique a aussi permis l'importation de nouveaux appareils photographiques et de caméras bon marché en comparaison du matériel surtaxé de la période précédente ${ }^{4}$. Ramana Hussain, Nalini Malani, Vivan Sundaram ou encore Najvot Altaf, sont parmi les premiers artistes à se tourner vers les installations, les vidéos et les performances, mais très peu d'espaces ont encore l'audace de présenter ces œuvres, dont les propos dénoncent souvent les traumatismes et les violences liées à la Partition et aux conflits politiques, religieux et sociaux récents.

En 1989, l'exposition Timeless Art, réalisée pour les 150 ans du Times of India, réunit les œuvres de la collection du puissant groupe de médias indiens. Il s'agit de la première grande vente aux enchères d'art moderne et contemporain organisée par Sotheby's, qui annonce une nouvelle phase dans l'apparition du marché de l'art indien.

Avec les réformes de Rajiv Gandhi en 1991, l'Inde s'ouvre pleinement au libéralisme, accélérant considérablement les transformations en cours dans le pays depuis l'Indépendance. Les grandes villes connaissent alors de profondes mutations et une expansion rapide. Les nouvelles mesures économiques permettent au secteur privé de se développer rapidement. Incarnant de nouvelles pratiques à la fois sociales, financières et individuelles, les directeurs de multinationales mais aussi une jeune génération qui a fait fortune dans le conseil, la gestion, l'informatique, constituent l'essentiel du nouveau public de collectionneurs et d'investisseurs qui participent au décollage du marché de l'art ${ }^{5}$. À l'échelle internationale, les Indiens de la diaspora ou 
NRIs (Non-Resident Indians) contribuent à l'élargissement de ce marché à l'étranger mais aussi à la reconnaissance et à la diffusion des artistes modernes et contemporains indiens. Parmi les quelque 500 collectionneurs sérieux que compte l'art contemporain indien, plusieurs sont d'origine étrangère: Charles Saatchi, le marchand d'art britannique d'origine iranienne, les Américains Chester et David Herwitz, le Japonais Masanori Fukuoka ${ }^{6}$ ou encore le Français François Pinault. Cependant, il faut attendre le début des années 2000 pour que, face à l'augmentation de la demande nationale et internationale, le marché de l'art s'organise professionnellement en Inde.

\section{Nouveaux territoires et économies de l'art dans les villes indiennes}

Les structures et les réseaux d'acteurs du marché de l'art, issus des mutations politiques et sociales des années 1950 et 1980, ont posé les bases de la réorganisation de l'activité artistique dans les villes indiennes. Dans quelle mesure celle-ci a-t-elle participé aux dynamiques urbaines et économiques? Comment a-t-elle permis à Delhi et à Mumbai de devenir de nouvelles centralités artistiques dans la géographie de l'art contemporain? L'évolution du rôle des galeries privées est l'un des enjeux majeurs, d'une part, dans l'émergence de nouveaux territoires et économies de l'art et, d'autre part, dans le renouvellement des pratiques et des circulations artistiques.

L'organisation actuelle des activités liées au marché de l'art en Inde montre une forte polarisation des deux métropoles internationales Delhi et Mumbai. Les galeries, au nombre d'une quinzaine dans chacune de ces deux villes avant 1990, se développent rapidement avec l'arrivée d'une clientèle indienne et internationale à partir des années 2000, avant de connaître en 2008 une période d'apogée puisqu'une cinquantaine de galeries sont alors présentes dans chaque ville. Il faut cependant relativiser ces chiffres dans la mesure où seule une minorité d'entre elles cherche réellement à accompagner de jeunes artistes dans leur carrière. Les plus ambitieuses ouvrent des succursales dans d'autres villes et à l'étranger, telles que Sakshi Gallery à Taipei ou The Guild à New York. Les maisons de ventes indiennes ont profité de cette effervescence artistique pour mettre en place un marché de l'art secondaire. La première, Osian, est fondée par Neville Tulli à Mumbai en 2000, suivie en 2003 par Saffronart, créée par Dinesh et Minal Vazirani. En inaugurant la première vente en ligne à l'automne 2008, le couple apporte un changement considérable au réseau du marché de l'art indien, attirant des acheteurs de trente-deux pays, dont la plupart d'Europe, de Chine et des États-Unis.

Kolkata (Calcutta), Bangalore ou Chennai, dont le développement artistique a suivi des trajectoires différentes, regroupent chacune une petite vingtaine de galeries. Kolkata, capitale intellectuelle et littéraire pendant la colonisation grâce au rayonnement de la famille Tagore puis Birla, n'est plus le centre artistique majeur qu'elle était, bien qu'elle possède encore plusieurs galeries importantes, dont CIMA (Centre of International Modern Art, Gallery Private Ltd) et la maison de ventes Emami Chisel Art, depuis 2008. Une situation qui va sans doute de pair avec son insertion économique plus lente dans le processus de mondialisation. À l'inverse, Bangalore incarne le décollage du secteur informatique et a su en quelques années attirer des groupes d'artistes, des galeries (SKE Gallery) et des résidences (1 Shanti Road, BAR 1) qui en font un nouveau pôle attractif et relativement proche de Mumbai. La maison de ventes Bid \& Hammer s'y est installée avec pour ambition de couvrir le marché du sud de l'Inde. Cette position est aussi 
convoitée par Chennai, l'autre capitale du sud, qui a lancé en 2011 sa première foire annuelle, Art Chennai, avec la participation des vingt-deux galeries de la ville. Baroda est restée un centre de production important, spécialement apprécié de la communauté artistique, offrant de nombreux studios et résidences, à seulement cinq heures de train de Mumbai. À Hyderabad, Kochi, Ahmedabad, Guwahati, plusieurs galeries essaient d'impulser de nouvelles dynamiques artistiques à l'échelle locale.

Figure 1

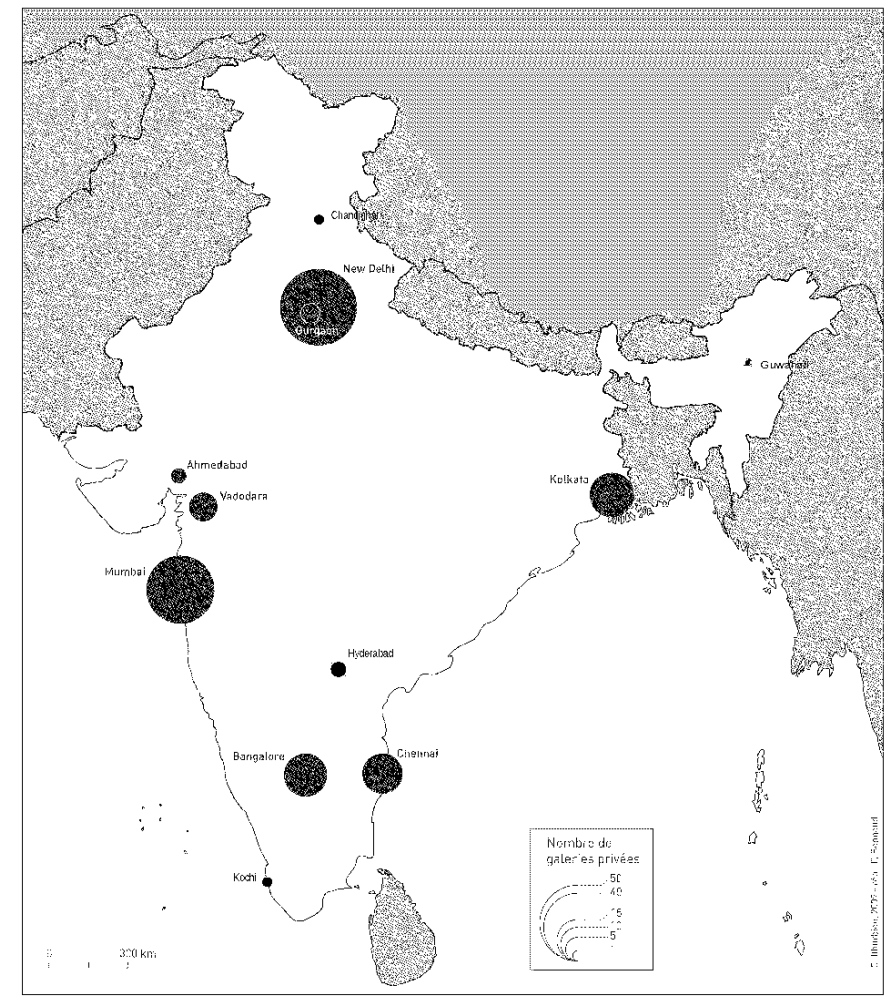

Les galeries publiques et privées d'art contemporain en Inde

L'absence de politique culturelle clairement écrite ${ }^{7}$ et de projet de planification artistique a donné lieu dans un premier temps à une organisation fragmentée et multipolaire des espaces artistiques à l'échelle des villes. La localisation des galeries, des studios et des fondations privées ont généralement suivi les contraintes liées à l'urbanisation et au prix du foncier. Le cas de Mumbai présente néanmoins le premier exemple d'émergence d'un "district artistique» en Inde et la construction de nouveaux rapports entre dynamiques urbaines, économiques et artistiques. La plupart des galeries sont rassemblées au sud de la ville, à Colaba, autour du centre historique Kala Ghoda, où sont aussi installés les deux principaux musées ${ }^{8}$ et la Jehangir Gallery. La renaissance de ce quartier est initiée par un groupe d'acteurs de la classe capitaliste transnationale - l'architecte Rahul Mehrotra, des journalistes, des urbanistes et des directeurs d'entreprises - préoccupés par la dégradation du patrimoine architectural de la ville. Afin de mobiliser des fonds pour la rénovation des bâtiments historiques, ils fondent la Kala Ghoda Association dont la mission est de revaloriser ce quartier et d'en faire un véritable Soho indien. Chaque année depuis 1999, l'association organise le Kala Ghoda Festival, devenu l'un des premiers grands festivals d'art dans l'espace public, et reçoit le soutien de nombreux mécènes privés. 
Progressivement, le quartier a connu une phase d'embellissement et d'embourgeoisement; il est désormais l'un des plus branchés et touristiques de la ville. Les galeries qui se sont peu à peu rapprochées de Colaba ont contribué aux transformations du quartier en termes d'image, d'espace mais aussi d'influence sur les pratiques artistiques. Dès 1999, les exemples de réhabilitation d'anciens locaux industriels se multiplient. Les directrices de Sakshi Gallery, Chemould Prescott Gallery ou encore The Guild, font appel à l'architecte Rahul Mehrotra pour convertir des bâtiments désaffectés en galeries d'art de standing international. Ces nouveaux lieux pouvaient dés lors proposer des œuvres de plus grandes dimensions et d'ambitieux projets d'installations, de vidéos et de performances. Maskara Gallery est sans doute celle qui a fait preuve des initiatives les plus marquantes de ces dernières années en accueillant l'impressionnante performance de Monali Meher, In determination II, sur les attentats de Mumbai de 2008, et les sculptures monumentales de T. Venkanna. Ces expositions à faible profit marchand contribuent néanmoins à valoriser le statut de la ville en tant que nouveau laboratoire de pratiques artistiques et curatoriales. Les galeries sont ainsi amenées à jouer un rôle significatif non seulement dans la remise en valeur de certains quartiers, mais elles ont aussi favorisé l'exploration des nouveaux médias et le rayonnement d'une jeune génération d'artistes indiens à l'étranger. Les galeries de Kala Ghoda-Colaba se sont imposées comme des espaces de référence du marché de l'art, incontournables et, par ailleurs, fréquemment sollicités lors d'expositions internationales. Beaucoup considèrent qu'elles ont remplacé l'État quant à son rôle dans l'ouverture de lieux d'art contemporain et dans la promotion et la diffusion des artistes. Alors qu'entre 1970 et 1989, les galeries privées produisaient moins d'un tiers des expositions de groupe indiennes, leur participation commence à s'accroître entre 1990 et 1999. De 2000 à 2005, elles en organisent presque la moitié et sont impliquées dans $75 \%$ des expositions organisées entre 2006 et $2010^{\circ}$.

À Delhi, les quelques galeries qui ont essayé de se regrouper pour former des villages d'art ou art street, à Haus Khas Village, dans les années 1990, puis à Lado Sarai depuis 2008 , n'ont pas réussi à créer des dynamiques économiques ou artistiques comparables. Historiquement, les premières galeries publiques et privées s'étaient installées dans les anciens quartiers de l'administration coloniale puis, entre 1960 et 1990, elles ont suivi le processus d'extension de la ville vers le sud et sa fragmentation en colonies d'habitation, souvent aménagées à l'intérieur de maisons privées ou sur les places commerciales. Néanmoins, l'absence de centralité artistique dans la capitale ne porte pas nécessairement préjudice au marché de l'art, dont une importante partie du chiffre d'affaires repose sur les transactions effectuées dans les hôtels de luxe, lors de préventes, ou sur des ventes en ligne ${ }^{10}$.

Si Mumbai et Delhi sont devenus des villes artistiques attractives, c'est en raison des initiatives et des collaborations récentes développées par les acteurs des réseaux marchands, alternatifs, non-gouvernementaux et privés qui s'y sont rassemblés. Les bénéfices générés par le marché de l'art ont commencé à favoriser une nouvelle industrie de l'art. Vadehra Art Gallery a ouvert la première librairie et bibliothèque d'art, Vadehra Book Store, et créé en 2008 une fondation pour l'art contemporain indien (FICA). L'augmentation rapide du nombre de catalogues d'expositions et de monographies consacrées à la scène artistique indienne a été stimulée par un nouveau cercle de lecteurs, des collectionneurs aux galeries, en comptant bien sûr les institutions indiennes et étrangères en demande de documentation scientifique. Un nouveau marché du livre d'art a émergé dans les villes et les revues en anglais se 
multiplient (Take on Art, Art Etc., Art Fair). De jeunes entrepreneurs dans le design et le graphisme travaillent pour les entreprises d'édition ou de communication et conçoivent de nouvelles gammes de produits (sacs, agendas, etc.), participant au développement d'une économie créative destinée à la classe moyenne et aisée urbaine. L'art contemporain et l'ensemble des activités qui y sont liées semblent alors se révéler progressivement comme un secteur jeune mais prometteur des industries culturelles indiennes.

Un tournant dans les politiques de l'État et des régions en faveur du secteur artistique semble s'amorcer. Du fait de l'intérêt grandissant du marché de l'art contemporain indien à l'échelle internationale, la Fédération indienne des chambres du commerce et de l'industrie (FICCI) a créé en 2009 une section «Art \& Business of Art $»^{11}$ qui a pour mission de mettre en place un nouvel agenda politique national pour l'industrie de l'art ("Art Industry ). L'ascension rapide de ce marché et de l'économie artistique qui lui est liée, semble avoir entraîné une nouvelle phase de développement de projets culturels où la tendance serait à la formation de partenariats public-privé et à l'instauration de politiques culturelles pour répondre aux besoins des métropoles.

\section{Mondialisation du marché de l'art, vers une scène artistique « indienne »}

Comment s'articulent les liens entre marché de l'art indien, promotion et diffusion des artistes indiens à l'échelle internationale? Quel fut le rôle des foires, des biennales et des grandes expositions à l'étranger dans l'institutionnalisation progressive de la scène indienne contemporaine? L'intérêt pour les artistes contemporains indiens se développe dans le contexte de la mondialisation de la culture et des arts. Alors que les études postcoloniales et les subaltern studies prennent de l'importance, de nouvelles voix contestent l'hégémonie euro-américaine et s'engagent dans une redéfinition des rapports centre/périphérie. Marché de l'art et institutions publiques s'intéressent alors à cette nouvelle géographie de l'art et multiplient les rétrospectives et les ventes annuelles concernant les pays émergents, symbolisant l'avènement d'un « Global art ».

Dès les années 1960, grâce à leurs voyages et parfois à leurs séjours de plusieurs années en Europe et aux États-Unis, les artistes indiens commencent à être exposés dans des galeries de Paris, de Londres ou de New York. C'est le cas pour Tyeb Mehta, S. H. Raza ou encore Akbar Padamsee. La plupart d'entre eux retourneront en Inde, forts d'un réseau international ${ }^{12}$. Peu de galeries installées à l'étranger sont alors consacrées à l'art contemporain d'Asie et encore moins à l'art contemporain indien. Elles ouvriront progressivement à New York (Sundaram Tagore) et à Londres (Grosvenor, Rossi \& Rossi). «La visibilité des artistes indiens à l'étranger a d'abord été marginale. Puis, l'accès aux plate-formes internationales fut réservé à ceux qui avaient été sélectionnés par le ICCR [Indian Council for Cultural Relations] ou la LKA [National Academy of Fine Art]. La plupart des artistes savaient que le marché international leur était quasi inaccessible, contrairement à ceux de la première génération d'artistes modernes $»^{13}$. À la fin des années 1970 et au début des années 1980, les festivals annuels et les expositions itinérantes - en France, en Angleterre, en Suisse mais aussi en Europe centrale (Belgrade, Prague et Bucarest) et au Japon - sont l'occasion d'organiser les premières expositions rétrospectives consacrées à des artistes indiens. 
L'art contemporain indien a pu intégrer ce système de production et de distribution globalisé de l'art contemporain parce qu'il a été porté par des réseaux d'acteurs transnationaux. Tout d'abord, les maisons de ventes internationales Sotheby's et Christie's lui ont permis de gagner une reconnaissance internationale en organisant des ventes annuelles d'art moderne et contemporain, à partir de 1995, principalement à Londres et à New York, où résident en majorité les Indiens de la diaspora. Le nombre de ventes annuelles a été multiplié par quatre, entre 2000 et $2005^{14}$, mais elles continuent à privilégier les artistes modernes qui représentent des valeurs sûres, en particulier depuis la crise économique de 2008.

Les plus grandes galeries indiennes commencent à participer aux foires d'art contemporain et permettent à une nouvelle génération d'acquérir une visibilité internationale. En 2009, l'Inde est le pays invité d'ARCO, la foire de Madrid. Les dix principales galeries de Delhi et Mumbai sont régulièrement présentes aux foires internationales (Art Basel, Art Miami, FIAC, Art Dubai), dont les ventes représentent pour certaines jusqu'à $50 \%$ de leur chiffre annuel ${ }^{15}$. Les biennales et les triennales sont aussi des événements importants dans la circulation des œuvres et des artistes indiens à l'étranger. Or, si, jusqu'au début des années 1990, la sélection des artistes et des commissaires participant à ces événements internationaux était encore principalement sous le contrôle de la LKA, des commissaires indépendants indiens, dont certains sont galeristes, prennent une place de plus en plus grande dans le processus de sélection et de diffusion ${ }^{16}$.

En parallèle, les musées internationaux commencent à faire l'acquisition d'œuvres d'artistes contemporains d'Asie (Moma, Tate Modern, Centre Pompidou, etc.). L'intérêt grandissant des commissaires d'expositions occidentaux pour la scène indienne favorise la reconnaissance des artistes dans les capitales internationales. Dans les années 1990, les expositions à l'étranger se multiplient et elles connaissent un véritable boom, à partir de $2005^{17}$, venant appuyer les propos d'Alain Quemin : « la validation par l'espace, par l'éloignement géographique a désormais remplacé la validation par le temps». Certaines de ces expositions n'auraient pas été possibles sans une collaboration avec les grandes galeries indiennes qui se sont parfois avérées être les principaux prêteurs ou financeurs d'œuvres conçues spécialement pour les expositions. Bien qu'il y ait des tentatives pour faire varier les approches conceptuelles, peu arrivent à se dégager d'une thématique avant tout nationale. Rares sont celles qui, à l'image de India Moderna, s'attachent à recontextualiser la création contemporaine dans sa complexité historique et artistique. Force est de constater également que, dans la plupart de ces blockbusters, la liste des artistes a bien peu changé, entre les années 2005 et 2011. Car il ne faut pas s'y tromper, comme le souligne Arshiya Lokhandwala, ces grandes expositions sont des références qui donnent à l'art indien la légitimité institutionnelle requise pour inciter son achat par les collectionneurs internationaux ${ }^{18}$. L'insertion de l'art contemporain indien dans le réseau des capitales artistiques internationales et l'intérêt qu'il a suscité à l'étranger auprès des espaces institutionnels et des marchés, ont contribué non seulement au développement de nouvelles dynamiques artistiques et économiques en Inde et en particulier dans les métropoles, mais ont aussi eu un impact sur les pratiques artistiques et curatoriales indiennes. 


\section{Conséquences sur les pratiques artistiques et curatoriales}

Le commissaire d'exposition (ou curateur) représente désormais un des critères internationaux de la production d'expositions. S'il existe depuis le début du xxe siècle de célèbres critiques et historiens de l'art en Inde ${ }^{19}$, le concept de commissaire d'exposition est beaucoup plus récent et cette profession ne s'est véritablement formalisée qu'à partir des années 1990. Dans les années 1970-1980, la frontière entre les statuts d'artiste, de professeur et de " commissaire d'exposition $»^{20}$ était très mince et ces responsabilités se cumulaient facilement, demandant à la fois une bonne connaissance des réseaux et de l'art. Ainsi, les artistes Krishen Kanna (1968) et Jyoti Bhatt (1975) ont été commissaires de la Triennale d'art de Delhi.

À partir des années 1990, un réseau de galeristes, de critiques d'art et de commissaires d'expositions indiens s'élabore dans les capitales artistiques et est indissociable de trois autres phénomènes, l'organisation du marché de l'art et des infrastructures publiques et l'émergence de la critique d'art. Les galeristes sollicitent des critiques d'art indépendants et les noms de Gayatri Sinha, Geeta Kapur ou Pooja Sud deviennent des références. Ranjit Hoskote, écrivain et poète, travaille en collaboration avec Pundole Art Gallery (Mumbai, 1994), The Guild (Mumbai, 2000), Sakshi (Mumbai, 2002), Ananth Art Gallery (Kolkata, 2006) mais aussi avec des institutions publiques indiennes et étrangères jusqu'à sa consécration en tant que commissaire du Pavillon indien à la Biennale de Venise en 2011. Ce phénomène se généralise aussi dans les espaces publics : Geeta Kapur est commissaire invitée de l'importante exposition One Hundred Years of Indian Art from the NGMA collection et Yashodhara Dalmia pour une rétrospective sur le Progressive Artists Group en 1996. Certains galeristes-collectionneurs prennent euxmêmes le rôle de commissaire pour des expositions aussi bien en Inde qu'à l'étranger. C'est le cas de Rakhi Sarkar (Singapour, 1997), Anupam Mehta, ou encore Shalini Shawney. Jusqu'ici, si de nombreux commissaires d'expositions indiens étaient formés sur le terrain, d'autres ont suivi des cursus à l'étranger comme Arshiya Lokhandwala ou Suman Gopinath, diplomées de Creative Curating au Goldsmiths College de Londres. Importées d'Occident, les premières formations en "art curating " ne sont apparues que depuis quelques années dans les écoles publiques (Jawaharlal Nehru University) et par l'intermédiaire de fondations privées (India Foundation for the Arts).

Avec l'arrivée de curateurs étrangers, intéressés pour exposer l'art contemporain indien dans leurs propres contextes, les critiques d'art-commissaires indiens commencent à être sollicités pour présenter cet art au niveau international. Cette ouverture implique l'insertion dans de nouveaux critères transculturels qui ont indirectement amené à un changement dans les concepts et le langage des jeunes $\operatorname{artistes}^{21}$. Dans leur ensemble, les expositions internationales ont largement favorisé les installations, les vidéos et les performances. Pratiquées par une minorité dans les années 1990, elles sont désormais largement répandues et expérimentées en Inde. L'intérêt pour ces nouveaux médias, correspondant aussi à un idéal postmoderne, a permis d'attirer l'attention sur des œuvres de grande qualité plastique et conceptuelle (Raqs Media Collective, Shilpa Gupta, Amar Kanwar). Cependant, la métamorphose rapide de quelques artistes en icônes de l'art contemporain indien a donné l'impression que ce type de production amènerait plus facilement à l'intégration et à la 
reconnaissance internationale. Les choix dans le soutien apporté par les galeries ont eux aussi été influencés par l'évolution de ce contexte transnational.

Partagés entre réappropriation du débat artistique et aspirations internationales, les artistes et les commissaires indiens cherchent à adopter certains codes ou modèles internationaux tout en développant des stratégies pour une expérience artistique plus locale. Les initiatives privées se multiplient pour remédier à l'absence de véritables infrastructures publiques pour l'art contemporain. $\mathrm{KHOJ}^{22}$ et SARAI ${ }^{23}$, souvent mentionnés, ont constitué une étape importante de la réinvention des pratiques curatoriales, notamment en faisant une place aux performances et aux installations dans l'espace public. De nouveaux lieux de production (Space 118, Creative Indian Foundation, What about art?, Asia Art Projects) et des résidences (Uttarayan Art Foundation), ouverts ces dernières années, répondent à une demande collective d'endroits adaptés aux besoins de la création contemporaine et pouvant soutenir les artistes dans des projets internationaux. Certains d'entre eux s'accompagnent désormais d'une progressive internationalisation des concepteurs, des conseillers artistiques, des ingénieurs techniques, des partenariats institutionnels et des filières de financement. Le faible intérêt des collectionneurs indiens pour l'art contemporain étranger a cependant freiné l'essor d'expositions d'artistes internationaux, même si récemment certaines galeries ont proposé une rétrospective de Wolfgang Laib (Chemould et Mirchandani + Steinruecke, Mumbai) et de Yoko Ono (Vadehra Gallery, Delhi). Face au manque de dynamisme dans les politiques d'acquisitions des collections publiques, les collectionneurs privés prennent une responsabilité particulière dans l'élaboration de nouvelles trajectoires et dans l'appréhension de l'évolution de la scène artistique. Toute discussion sur les pratiques curatoriales en Inde ne peut cependant faire abstraction du déséquilibre dans le développement des institutions artistiques publiques et privées, les initiatives et les financements privés étant actuellement beaucoup plus nombreux ${ }^{24}$, rappelle Jyoti Dhar.

\section{Conclusion}

L'art contemporain est devenu l'un des nouveaux emblèmes de la modernité culturelle des métropoles indiennes. L'émergence de nouveaux lieux d'art et d'événements artistiques, qui s'inscrit dans le contexte de mondialisation des villes, laisse apparaître une volonté grandissante d'insertion dans le réseau des capitales culturelles internationales. Les enjeux de l'évolution des dynamiques du marché de l'art s'observent à plusieurs échelles. À celle des villes, de nouveaux territoires urbains de l'art contemporain se mettent en place, prenant part, entre autres, à des phénomènes d'embourgeoisement, d'embellissement et de développement de nouvelles industries culturelles. Si l'espace artistique urbain s'est progressivement réorganisé autour des structures du marché de l'art, en particulier des galeries, remplissant des fonctions symboliques, économiques et sociales singulières, il reste difficile de parler de « districts artistiques » en Inde, sauf dans certains cas très particuliers. À l'échelle nationale, l'organisation polycentrique s'affirme. Même si Delhi et Mumbai continuent de dominer la scène artistique, les pôles secondaires cherchent de nouveaux échanges et stratégies de visibilité pour attirer davantage d'artistes, de collectionneurs, de critiques, de visiteurs locaux et internationaux. De nombreuses initiatives, de construction de musées (KMoMA à Kolkata), de lancement de biennales (Kochi-Muziris, décembre 2012), témoignent d'une volonté de s'insérer dans ce réseau de l'art et de 
bénéficier de l'attractivité territoriale générée par le nouvel engouement pour l'art contemporain. À l'échelle internationale, l'intérêt de la diaspora, des commissaires d'expositions et du public a aussi eu des répercussions sur le développement de cette activité, dans un contexte où les frontières entre les intérêts commerciaux et scientifiques sont de plus en plus minces. Enfin, s'il manque en effet des infrastructures publiques permettant l'élaboration de réflexions durables sur l'art, indépendantes des intérêts et des fluctuations du marché, l'État doit aussi s'interroger sur les barrières créées par les lois actuelles sur les donations ou sur la taxation à l'importation et à la circulation des œuvres à la fois entre ses États fédéraux et avec l'international. Ces revendications des professionnels du milieu de l'art contemporain indien et étranger se font désormais largement entendre et pourraient ouvrir sur de nouvelles possibilités d'échanges et de développement artistique.

\section{NOTES}

1. Parmi les écoles d'art, l'Academy of Fine Arts de Calcutta, la J. J. School of Art à Bombay; les musées Indian Museum à Calcutta, le Victoria \& Albert Museum à Bombay (renommé Bhau Daji Lad Museum) et la société artistique, Bombay Art Society.

2. En 1995, la ville de Bombay est renommée Mumbai par le Siv Sena, le parti régionaliste Marâthî à la tête de la municipalité. Les deux noms seront utilisés en fonction du contexte historique auquel ils font référence.

3. Pour plus de détails, voir Christine Ithurbide, "Les femmes entrepreneurs dans l'art contemporain en Inde ", in actes du colloque Femmes au pouvoir, Paris-I Sorbonne, Paris, juin 2011, à paraître.

4. Voir Devika Singh « Contextualiser l'art contemporain indien. Une histoire des expositions de groupe de 1968 à nos jours ", catalogue de l'exposition Paris, Delhi, Bombay..., Centre Pompidou, Paris, 2011.

5. Voir l'article de Mila Gantcheva, "Les très riches heures de la peinture indienne ", Le Monde diplomatique, $\mathrm{n}^{\circ}$ 94, 2007 : 90-93.

6. Dans le Royal Academy magazine, 2006: http://www.royalacademy.org.uk/ra-magazine/ winter2006/features/made-in-india,49,RAMA.html

7. Propos d'Ashok Vajpeyi, lors de la conférence Made in Delhi : Post-1947 Cultural Institutions, SARAI, Delhi, 13 avril 2011.

8. National Gallery of Modern Art et Chatrapati Shivaji Maharaj Vastu Sangrahalaya (ancien Prince of Wales Museums).

9. Pourcentage d'expositions de groupe ( 3 artistes indiens minimum) organisées par des galeries privées : $19 \%$ entre 1970 et $1979 ; 18 \%$ entre 1980 et $1989 ; 31 \%$ entre 1990 et $1999 ; 47 \%$ entre 2000 et $2005 ; 72 \%$ entre 2006 et 2010. Ces statistiques ont été réalisées à partir de la chronologie produite par Évelyne Pomey, voir le catalogue de l'exposition Paris, Delhi, Bombay..., op.cit. : 228-257.

10. Pour plus de détails, voir Christine Ithurbide, « Le marché de l'art contemporain indien : enjeux de la mondialisation », Géographie et culture, $n^{\circ} 74-75,2010: 207-228$. 
11. Voir aussi le rapport de Mousumi Ghose, "Art Industry in India », FICCI, 2010, fiche de présentation en ligne consultée le 28 juillet 2012. URL:http://www.ficci.com/publicationpage.asp?spid=20055

12. Devika Singh, dans le catalogue de l'exposition Paris, Delhi, Bombay..., op. cit. : 89.

13. Gulam Mohammed Sheik interwievé par Akansha Rastogi dans Art \& Deal, vol. $7, n^{\circ} 2$, issue 32 :38-47.

14. Alors qu'une seule vente annuelle est organisée entre 2000 et 2004 , les deux maisons de ventes dédient depuis 2005 quatre ventes aux artistes modernes et contemporains indiens. Elles ont lieu à Londres, à New York, à Singapour, à Dubaï ou encore à Hongkong, là où résidennt par ailleurs de nombreux NRIs.

15. Interview de Chemould Prescott Gallery dans Take On Art, vol. 2, issue 1, avril 2011.

16. Tate Modern podcast «Mapping Mumbai» 1: www.planetizen.com/audio/by/title/ planetizen_podcast_-_2007-11-16_-

_bombay_to_mumbai_a_megacity_in_the_developing_world_-_part_one

17. Edge of Desire : Recent Art in India en 2005 à New York; Horn Please en 2006 en Allemagne ; Indian Summer en 2005 à Paris ; Mumbai Maximum City à Lille en 2006 ; Indian Highway en 2008 à Londres ; Chalo India! en 2009 à Tokyo; India Moderna en 2009 à Valence; The Empire Strikes Back: Indian art today en 2010 à Londres; Delhi, Bombay... au Centre Pompidou à Paris en 2011.

18. Voir Take On Art, vol. 2, issue 1, avril $2011: 22$.

19. Ananda Coomaraswamy (1877-1947), Bishnu De (1909-1982), Mulk Raj Anand (1905-2004) et Richard Bartolomew (1926-1985).

20. Ce n'est qu'à partir de 1994 que le terme « conservateur » est utilisé en Inde, voir Take On Art, vol. 2, n 1, avril 2011.

21. Geeta Kapur interwievée par Natasha Ginwala dans Art \& Deal, vol. 7, $n^{\circ} 2$, issue $32: 20-36$.

22. Voir par exemple le festival $48^{\circ} \mathrm{C}$ Public.Art.Ecology (2008) et le Yamuna-Elbe Project (2011).

23. Khoj est un espace atelier et une résidence pour artistes, basé à Delhi et Sarai est un espace de recherche, d'essais et d'échanges sur les médias contemprorains et les constallations urbaines $(\mathrm{NdR})$

24. Jyoti Dhar, "Curatorial Practice inIndia: between the alternative and the mainstream ", ArtAsiaPacifi, issue 76, novembre-décembre 2011: 56-57.

\section{RÉSUMÉS}

Cet article propose d'analyser l'émergence du marché de l'art contemporain indien, ses enjeux dans les dynamiques urbaines et économiques des métropoles indiennes et dans la transformation des pratiques et discours en Inde sur l'art contemporain. De l'Indépendance à la fin des années 1980, le réseau d'espaces artistiques en Inde ne présente qu'un petit nombre de galeries publiques et privées dans les grandes villes (Delhi, Bombay, Calcutta, Madras) et plusieurs écoles artistiques régionales importantes (Baroda, Santiniketan). La libéralisation édconomique du pays, accélérée par les réfomres de 1991, entraîne une rapide multiplication des galeries privées et de la progressive professionnalisation du marché de l'art. Delhi et Mumbai émergent rapidement comme les centres de l'art comtemporain indien.L'ensembl edes activités liées au marché de l'art (production, édition, investissements) participe à la création de nouveaux territoires et éconimies de l'art et contribue au statut de capitale culturelle 
mondialisée. Si les structures de marché de l'art dominent dans les métropoles, l'ouverture de lieux alternatifs, résidences et collectifs, permettent à de nouvelles pratiques et discours de se développer. Sur les modèles internationaux, certaines villes tentent d'établir des quartiers artistiques, mais l'absence de soutien de l'État et de politiques publiques pour le développement des arts ont davantage entraîné une organisation fragmentée et multipolaire des espaces artistiques. Les iniatiatives et financements du secteur privé prennent une importance particuli ère dans la trajectoire prise par la scène artistique indienne. Par ailleurs, l'émergence du marché de l'art contemporain indien s'inscrit dans une période d'avènement de l'art global et des redéfinitions des rapports de pouvoir entre les pays dits «du centre» et ceux de la "périphérie ». Avec la multiplication des expositions itinérantes et l'engouement pour la scène artistique indienne, les pratiques artistiques et curatoriales s'adaptent à ce nouveau contexte. Les foires, biennales, grandes expositions à l'étranger ont ainsi progressivement institutionnalisé la scène indienne. Partagés entre réappropriation du débat artistique et aspirations internationales, les artistes, galieristes et commissaires indiens adoptent certains codes ou modèlent et cherchent à renouveler l'approche des arts visuels à partir de contextes culturels très variés. Si la création artistique contemporaine indienne a désormais intégré le système d'expositions et de ventes internationales, elle est aussi devenue fortement dépendante de la pression du marché et des modèles internationaux. Au-delà du maintien de cette visibilité internationale, la question de l'insertion dans des dynamqiues régionales durables et de la construction d'un véritable dialogue avec une audience locale, sera aussi essentielle pour son avenir.

This article propose to analyze the emergence of Indian contemporary art market, its roles in economic and urban dynamics of indian cities and in the transformation of practices and discourses on India art. From the Independence to the late 1980s, the network of visual art spaces in India provides only a few public and private galleries in major cities (Delhi, Bombay, Calcutta, Madras) and several major regional art schools (Baroda, Santiniketan). Accelerated by the reforms of 1991,The country's economic liberalization leads to rapid proliferation of private galleries and the gradual professionalization of the art market. Delhi and Mumbai are rapidly emerging as centers of Indian contemporary art. Activities related to the art market (production, publishing, investment) participate to the creation of new art territories and economies and contribute to the status of global cultural capital. If market structures dominate in the cities, the creation of alternative spaces, residences and artists collective enable the development of new experiences and debates. Following international models, some neighborhoods attempt to become art districts. However the lack of State support and public policy for the development of the arts have led to a rather segmented organization of art spaces. Private sector initiatives and funding continue to play an important role in the trajectory taken by the Indian art scene. Moreover, the emergence of Indian contemporary art market occur during a period of reflexion on global art and redefinition of power relations between the countries so-called "center" and "periphery." With the multiplication of exhibitions and the interest for the Indian art scene, artistic and curatorial practices have adapted to this new context. Fairs, biennials, travelling exhibitions have contributed to the gradually institutionalization of the Indian scene. Shared between the reappropriation of artistic debate and international aspirations, artists, gallery owners and curators would adopt certain codes or models and try to renew the approach of visual arts from varied cultural learnongs and traditions. If the Indian contemporary art has now integrated international exhibitions and auctions, it has also become highly dependent on market pressures and international models. Beyond the maintenance of this global exposure, the question of integration into regional contexts and long-term construction of a genuine dialogue with a local audience, will also be essential for its future. 
INDEX

Mots-clés : art contemporain, Inde, marché de l'art, réseaux artistiques, villes mondialisées

Keywords : contemporary art, India, art market, artistic networks, global cities

\section{AUTEUR}

\section{CHRISTINE ITHURBIDE}

Université Paris-VII, Unité mixte de recherche (UMR) 7135, Sociétés en développement, études transdisciplinaires (SEDET)

c.ithurbide@orange.fr 